\title{
Sedentary Behavior, Chronic Diseases, and COVID-19
}

\author{
Young Gyu Cho* \\ Department of Family Medicine, Inje University Seoul Paik Hospital, Inje University College of Medicine, Seoul, Korea
}

\section{See original paper on 27}

Sedentary behavior refers to any waking behavior, such as using an electronic device, reading a book, or sitting in a car, with an energy expenditure of 1.5 metabolic equivalent for the task or less, while in a sitting, reclining, or lying position. ${ }^{1)}$ Sedentary behavior is distinct from physical inactivity, which is defined as an insufficient level of physical activity to meet present recommendations. ${ }^{2)}$ Individuals who are considered physically active under current guidelines may spend most of their waking time in sedentary behaviors. ${ }^{3)}$ Prolonged, uninterrupted sedentary behavior is a modifiable risk factor for chronic diseases independent of physical activity level. ${ }^{4)}$ The World Health Organization presented moderate evidence that a high level of sedentary behavior is associated with an increased risk of mortality from all causes, cardiovascular disease (CVD), and cancer, and the incidence of type 2 diabetes, CVD, and cancer. ${ }^{5)}$ In the present issue, Concha and Mendo$\mathrm{za}^{6)}$ reported that sedentary behavior was associated with the development of chronic kidney disease in most of the studies included in their systematic review.

Since the outbreak of coronavirus disease 2019 (COVID-19), most countries around the world have adopted social distancing measures to reduce transmission of COVID-19. ${ }^{7)}$ The Korean government has also implemented a variety of measures to encourage staying at home, limit non-essential gatherings, and promote remote work and online learning. ${ }^{8}$ These measures may result in changes in daily lifestyle, including physical activity and sedentary behavior. Stockwell et al. ${ }^{9)}$ demonstrated that physical activity decreased with concurrent increases in sedentary behavior during the lockdown due to the COVID-19 pandemic, irrespective of subpopulation. These findings were also consistently found in studies conducted in
Korea. $^{10,11)}$ Prolonged sedentary behavior during the COVID-19 pandemic may increase cardiometabolic risks and potentially exacerbate underlying chronic diseases. ${ }^{12)}$ A Korean study revealed that change in body mass index (BMI) during COVID-19 pandemic was inversely associated with change in physical activity and positively associated with change in sedentary time. ${ }^{11)}$ In a retrospective cohort study conducted at a hospital in Korea, cardiometabolic risks including BMI, blood pressure, lipid profiles, and glycated hemoglobin worsened, and the proportion of patients with metabolic syndrome significantly increased during the COVID-19 pandemic. $^{13)}$

Given that the COVID-19 pandemic has been prolonged over a couple of years, the negative impact of COVID-19-related social distancing measures on health behaviors and chronic diseases is expected to be more serious. Sedentary behavior is recommended as a target to counter the negative health consequences of social restrictions. Interruption to sedentary behavior at home or at work can be achieved without increased risk of COVID-19 infection. Standing and walking for 2-5 minutes every 20-30 minutes of sedentary behavior can be effective in reducing the risk of chronic diseases. ${ }^{14)}$ Ricci et al. ${ }^{15)}$ proposed general recommendations to stay active and limit sedentary behavior during the COVID-19 pandemic. Policymakers and health professionals should provide and encourage practical strategies to reduce and interrupt sedentary behavior while implementing COVID-19-related social distancing measures.

\section{CONFLICT OF INTEREST}

No potential conflict of interest relevant to this article was reported. 


\section{ORCID}

Young Gyu Cho: https://orcid.org/0000-0003-1017-8884

\section{REFERENCES}

1. Tremblay MS, Aubert S, Barnes JD, Saunders TJ, Carson V, LatimerCheung AE, et al. Sedentary Behavior Research Network (SBRN): Terminology Consensus Project process and outcome. Int J Behav Nutr Phys Act 2017;14:75.

2. Panahi S, Tremblay A. Sedentariness and health: is sedentary behavior more than just physical inactivity? Front Public Health 2018;6:258.

3. Dunstan DW, Dogra S, Carter SE, Owen N. Sit less and move more for cardiovascular health: emerging insights and opportunities. Nat Rev Cardiol 2021;18:637-48.

4. Melo EA, Ferreira LE, Cavalcanti RJ, Botelho Filho CA, Lopes MR, Barbosa RH. Nuances between sedentary behavior and physical inactivity: cardiometabolic effects and cardiovascular risk. Rev Assoc Med Bras (1992) 2021;67:335-43.

5. Dempsey PC, Biddle SJ, Buman MP, Chastin S, Ekelund U, Friedenreich $\mathrm{CM}$, et al. New global guidelines on sedentary behaviour and health for adults: broadening the behavioural targets. Int J Behav Nutr Phys Act 2020;17:151.

6. Concha AT, Mendoza FA. Sedentarism, a modifiable risk factor for developing chronic kidney disease in healthy people. Korean J Fam Med 2022;43:27-36.

7. Chiesa V, Antony G, Wismar M, Rechel B. COVID-19 pandemic: health impact of staying at home, social distancing and 'lockdown' measures: a systematic review of systematic reviews. J Public Health (Oxf) 2021;43:e462-81.

8. Government of the Republic of Korea. All about Korea's response to COVID-19 [Internet]. Seoul: Ministry of Foreign Affairs; 2020 [cited 2022 Jan 5]. Available from: http://ncov.mohw.go.kr/upload/ncov/file /202010/1602724510959_20201015101517.pdf.

9. Stockwell S, Trott M, Tully M, Shin J, Barnett Y, Butler L, et al. Changes in physical activity and sedentary behaviours from before to during the COVID-19 pandemic lockdown: a systematic review. BMJ Open Sport Exerc Med 2021;7:e000960.

10. Park KH, Kim AR, Yang MA, Lim SJ, Park JH. Impact of the COVID-19 pandemic on the lifestyle, mental health, and quality of life of adults in South Korea. PLoS One 2021;16:e0247970.

11. Kim GH, Yun HW, Cho W, Kimm H, Jung KJ, Jee SH. Relationship between changes in sedentary time, physical activity, and body mass index during the COVID-19 pandemic in Korea. Asia Pac J Public Health 2021;33:958-60.

12. Chandrasekaran B, Ganesan TB. Sedentarism and chronic disease risk in COVID 19 lockdown: a scoping review. Scott Med J 2021;66:3-10.

13. Sohn M, Koo BK, Yoon HI, Song KH, Kim ES, Kim HB, et al. Impact of COVID-19 and associated preventive measures on cardiometabolic risk factors in South Korea. J Obes Metab Syndr 2021;30:248-60.

14. Zieff G, Bates LC, Kerr ZY, Moore JB, Hanson ED, Battaglini C, et al. Targeting sedentary behavior as a feasible health strategy during COVID-19. Transl Behav Med 2021;11:826-31.

15. Ricci F, Izzicupo P, Moscucci F, Sciomer S, Maffei S, Di Baldassarre A, et al. Recommendations for physical inactivity and sedentary behavior during the coronavirus disease (COVID-19) pandemic. Front Public Health. 2020 May 12;8:199. 